\title{
THE RECIPROCAL OF A REAL ENTIRE FUNCTION AND NON-REAL ZEROS OF HIGHER DERIVATIVES
}

\author{
James K. Langley \\ University of Nottingham, School of Mathematical Sciences \\ Nottingham, NG7 2RD, United Kingdom; jkl@maths.nott.ac.uk
}

\begin{abstract}
Two theorems are proved concerning non-real zeros of derivatives of the reciprocal of a real entire function with real zeros. A further result treats the frequency of non-real poles for real meromorphic functions which together with their first three derivatives have only real zeros.
\end{abstract}

\section{Introduction}

This paper concerns non-real zeros of derivatives of real meromorphic functions in the plane, that is, meromorphic functions mapping $\mathbf{R}$ into $\mathbf{R} \cup\{\infty\}$. Extensive research into the non-real zeros of derivatives of real entire functions $[2,3,4,5,7$, $16,17,23,24,25,35,38$ ] arose largely from the conjecture of Wiman (now proved in $[3,35,38])$ that if $f$ is a real entire function such that $f$ and $f^{\prime \prime}$ have only real zeros, then $f$ belongs to the Laguerre-Pólya class consisting of locally uniform limits of real polynomials with real zeros, as well as from related conjectures of Pólya [36]. The starting point of the present paper is the analogous problem where $f$, rather than being entire, is the reciprocal of a real entire function with real zeros. The following theorem [31, 32] represents a strengthening of results of Hellerstein and Williamson [18] and Rossi [37]. Here $N_{N R}(r, g)$ denotes the Nevanlinna counting function [12] of the non-real poles of a meromorphic function $g$ in the plane, and this notation will be used throughout the paper.

Theorem 1.1. [31, 32] Let $f$ be a real meromorphic function in the plane, with finitely many zeros and non-real poles, but not of the form

$$
f=S e^{P} \text {, with } S \text { a rational function and } P \text { a polynomial. }
$$

Then $f^{\prime \prime}$ has infinitely many non-real zeros. If, in addition, $f$ has finite lower order, then $N_{N R}\left(r, 1 / f^{\prime \prime}\right) \neq o\left(T\left(r, f^{\prime} / f\right)\right)$ as $r \rightarrow \infty$.

The author conjectures that if $f$ is as in the hypotheses of Theorem 1.1 then $f^{(k)}$ has infinitely many non-real zeros for every $k \geq 2$. The proof of Theorem 1.1 was accomplished in two stages, using different methods, depending on the growth of $f$. For the case where $f$ is a real meromorphic function of finite lower order in the plane, with finitely many zeros and non-real poles, the key step was to show that $f^{\prime \prime}$ has finitely many real zeros [31], a result which is not true for infinite lower order as the example $f(z)=\exp (\sin z)$ immediately shows. The assertion of Theorem 1.1 for the case of finite lower order was then proved in [31] by applying a theorem of Eremenko [8]. The main result of the present paper will again treat the case where $f$

doi:10.5186/aasfm.2013.3847

2010 Mathematics Subject Classification: Primary 30D20, 30D35.

Key words: Meromorphic function, non-real zeros. 
has finite lower order, to give a counterpart of Theorem 1.1 for the third and fourth derivatives, as well as to strengthen the previous result for the second derivative.

Theorem 1.2. Let $f$ be a real meromorphic function of finite lower order in the plane, with finitely many zeros and non-real poles, not of the form (1). Then $f$ satisfies, as $r \rightarrow \infty$ in a set of logarithmic density 1 ,

$$
T\left(r, f^{\prime} / f\right) \sim \frac{1}{2} N_{N R}\left(r, 1 / f^{\prime \prime}\right) \sim \frac{1}{2} N_{N R}\left(r, 1 / f^{(3)}\right) \sim \frac{1}{4} N_{N R}\left(r, 1 / f^{(4)}\right) .
$$

Thus, under the hypotheses of Theorem 1.2 , each of $f^{\prime \prime}, f^{(3)}$ and $f^{(4)}$ has infinitely many non-real zeros. On the other hand, Lemma 5.3 below shows that most of these zeros lie relatively close to the real axis. The strategy for proving Theorem 1.2 is to demonstrate (see Proposition 5.1 below) that if $f$ is as in the hypotheses then $f^{(4)}(x) / f(x)$ is positive or infinite for all real $x$ with $|x|$ sufficiently large, as was already proved for $f^{\prime \prime}(x) / f(x)$ in [31]. Indeed, it seems reasonable to conjecture that the same would be true for $f^{(n)}(x) / f(x)$, for any even positive integer $n$, but this appears to be difficult to prove.

For functions of infinite order the following theorem provides a partial resolution of the conjecture raised following Theorem 1.1. The case $m=0$ is already contained in Theorem 1.1, but the present proof is somewhat simpler than that in [32].

Theorem 1.3. Let $f$ be a real meromorphic function of infinite order in the plane, with finitely many zeros and non-real poles. Let $m$ be a non-negative integer. Then at least one of $f^{(m)}$ and $f^{(m+2)}$ has infinitely many non-real zeros.

The final result is linked to the investigations of [28, 30, 31, 32, 33], which in turn followed on from earlier work [18, 19, 20, 21, 22, 37] concerning the existence of non-real zeros of derivatives of real meromorphic functions in general. It seems likely that if $k \geq 2$ and $f$ is a real meromorphic function in the plane, such that $f$ and $f^{(k)}$ have finitely many non-real zeros, then $f$ has in some sense relatively few distinct non-real poles. A partial result in this spirit holds if in addition the non-real poles of $f$ have bounded multiplicities, in which case there exists $B>0$ such that if $z_{0}$ and $z_{1}$ are distinct non-real poles of $f$ then $\left|z_{1}-z_{0}\right|>B\left|\operatorname{Im} z_{0}\right|$ (see [33, Lemma 4.3]). The following theorem from [33] showed that if $k=2$ and $f^{\prime} / f$ has finite lower order, this additional hypothesis on the multiplicities of non-real poles is not required.

Theorem 1.4. [33] Let $f$ be a real meromorphic function in the plane such that $f^{\prime} / f$ has finite lower order and $f$ and $f^{\prime \prime}$ have finitely many non-real zeros. If $z_{0}$ is a non-real pole of $f$ and $\left|z_{0}\right|$ is sufficiently large then $f$ has no poles in the set

$$
\left\{z \in \mathbf{C}: 0<\left|z-z_{0}\right|<\frac{\left|\operatorname{Im} z_{0}\right|}{16}\right\} \text {. }
$$

In the next result the assumption that the logarithmic derivative has finite lower order is dispensed with, at the cost of considering more derivatives.

Theorem 1.5. Let $n$ and $m$ be non-negative integers with $n \notin\{m, m+1, m+2\}$. Let $g$ be a real meromorphic function in the plane such that $g^{(n)}, g^{(m)}, g^{(m+1)}$ and $g^{(m+2)}$ all have finitely many non-real zeros. If $z_{0}$ is a non-real pole of $g$ and $\left|z_{0}\right|$ is sufficiently large then $g$ has no poles in the set (3).

Theorems 1.4 and 1.5 imply that if $0<\beta<\pi / 2$ then the number of distinct poles with $|z| \leq r, \beta \leq|\arg z| \leq \pi-\beta$ is $O(\log r)$ as $r \rightarrow \infty$. In particular this holds 
for a real meromorphic function $f$ such that $f, f^{\prime}, f^{\prime \prime}$ and $f^{\prime \prime \prime}$ have finitely many non-real zeros, with no assumption on the growth of $f$ or the multiplicities of poles. However, this seems unlikely to be sharp.

\section{Direct singularities and the Tsuji characteristic}

Let $a \in \mathbf{C}$ be an asymptotic value of a transcendental meromorphic function $F$ in the plane, so that $F(z) \rightarrow a$ as $z \rightarrow \infty$ along a path $\gamma$. Then the inverse function $F^{-1}$ is said to have a transcendental singularity over $a$ [1]. For each $\varepsilon>0$ there then exists a component $C=C(a, \varepsilon, F)$ of the set $\{z \in \mathbf{C}:|F(z)-a|<\varepsilon\}$ such that $C$ contains an unbounded subpath of $\gamma$. Two asymptotic paths $\gamma, \gamma^{\prime}$ on which $F(z) \rightarrow a$ determine distinct singularities if the corresponding components $C(a, \varepsilon, F), C^{\prime}(a, \varepsilon, F)$ are disjoint for some $\varepsilon>0$.

The singularity of $F^{-1}$ corresponding to $\gamma$ is called indirect [1] if $C(a, \varepsilon, F)$, for every $\varepsilon>0$, contains infinitely many zeros of $F-a$, and direct otherwise. A direct singularity will be referred to as lying in the open upper half-plane $H=\{z \in$ C: $\operatorname{Im} z>0\}$ if $C(a, \varepsilon, F) \subseteq H$ for sufficiently small positive $\varepsilon$. Transcendental singularities over $\infty$ are defined and classified analogously.

The following lemma from [29] established a link between the number of direct singularities lying in the open upper half-plane $H$ and the growth of the Tsuji characteristic $\mathfrak{T}(r, G)$ [39], which is defined for functions $G$ which are meromorphic in the closed upper half-plane $\bar{H}=\{z \in \mathbf{C}: \operatorname{Im} z \geq 0\}$ (see also [11]).

Lemma 2.1. [29] Let $G$ be a meromorphic function in the plane such that $\mathfrak{T}(r, G)=O(\log r)$ as $r \rightarrow \infty$. Then there is at most one direct singularity of $G^{-1}$ lying in $H$.

Lemma 2.2. Let $L \not \equiv 0$ be a real meromorphic function in the plane such that $\mathfrak{T}(r, L)=O(\log r)$ as $r \rightarrow \infty$, and define $F$ by $F(z)=z-1 / L(z)$. Assume that at least one of $L$ and $1 / L$ has finitely many non-real poles. Then there exist finitely many $\alpha \in \mathbf{C}$ such that $F(z)$ or $L(z)$ tends to $\alpha$ as $z$ tends to infinity along a path in $\mathbf{C} \backslash \mathbf{R}$.

Proof. Suppose first that $1 / L$ has finitely many non-real poles: then so has $F$. Assuming that the assertion of the lemma is false leads to the following, in which $G$ is $F$ or $1 / L$ and so satisfies $\mathfrak{T}(r, G)=O(\log r)$ as $r \rightarrow \infty$. There exist, in view of the fact that $G$ is real, pairwise distinct $\alpha_{1}, \alpha_{2}, \alpha_{3}$ in $\mathbf{C}$ such that $G(z)$ tends to $\alpha_{p}$ as $z \rightarrow \infty$ on a path $\gamma_{p}$ in $H$. But this gives at least two direct singularities of $G^{-1}$ over $\infty$, lying in $H$, contradicting Lemma 2.1.

Now assume that $L$ has finitely many non-real poles. Then the above argument delivers the conclusion of the lemma for both $L$ and the function $M$ defined as in [3] by

$$
M(z)=z^{2} L(z)-z=\frac{z F(z)}{z-F(z)} .
$$

Following [3], if $F(z)$ tends to $\alpha \in \mathbf{C}$ as $z$ tends to infinity along a path, then so does $M(z)$, and this completes the proof. 


\section{Tools from the Wiman-Valiron theory}

Lemma 3.1. [14] Let the function $P$ be transcendental and meromorphic with finitely many poles in the plane, and let $q \in \mathbf{N}$. Then there exists a set $E_{1}$ of finite logarithmic measure such that, if $r \in[1, \infty) \backslash E_{1}$ and $\left|z_{1}\right|=r,\left|P\left(z_{1}\right)\right| \sim M(r, P)=$ $\max \{|P(z)|:|z|=r\}$ then

$$
P(z) \sim P\left(z_{1}\right)\left(\frac{z}{z_{1}}\right)^{N(r)} \text { and } \frac{P^{(j)}(z)}{P(z)} \sim \frac{N(r)^{j}}{z^{j}} \quad \text { for } \quad\left|\log \frac{z}{z_{1}}\right| \leq N(r)^{-7 / 12}
$$

and $j=1, \ldots, q$. Here $N(r)$ tends to infinity with

$$
N(r)=O(\log M(r, P))^{2} \quad \text { for } \quad r \in E_{1} .
$$

Proof. When $P$ is entire these are standard facts from the Wiman-Valiron theory [14], with $N=N(r)$ the central index of $P$. In the general case let $w_{1}, \ldots, w_{m}$ be the poles of $P$, repeated according to multiplicity. Then the assertions of the lemma follow from Leibnitz' rule and the corresponding estimates for the entire function $P_{1}(z)=P(z) \prod_{1 \leq k \leq m}\left(z-w_{k}\right)$. With a slight abuse of notation, $N(r)$ may still be referred to as the central index of $P$.

\section{Further lemmas required for Theorems 1.2 and 1.3}

Lemma 4.1. There exists a positive real number $\varepsilon$ with the following property. There do not exist positive real numbers $A, B, C, D, E$ satisfying

$$
E^{4} A^{4}+6 E^{3} A^{2} B+3 E^{2} B^{2}+E D<(4+\varepsilon) E^{2} A C \quad \text { and } \quad C^{2}<\frac{3 B D}{4} .
$$

Proof. Assume that $\varepsilon$ is small and positive and that positive real numbers $A, B, C, D, E$ satisfy both inequalities in (5), so that in particular

$$
D<(4+\varepsilon) E A C<(4+\varepsilon) E A \sqrt{\frac{3 B D}{4}}, \quad \sqrt{D}<(4+\varepsilon) E A \sqrt{\frac{3 B}{4}} .
$$

On substitution into (5) this yields

$$
\begin{aligned}
E^{4} A^{4}+6 E^{3} A^{2} B+3 E^{2} B^{2}+E D & <(4+\varepsilon) E^{2} A \sqrt{\frac{3 B}{4}} \cdot(4+\varepsilon) E A \sqrt{\frac{3 B}{4}} \\
& =\frac{3}{4}(4+\varepsilon)^{2} E^{3} A^{2} B .
\end{aligned}
$$

On the other hand (5) also leads to

$$
6 E^{3} A^{2} B<(4+\varepsilon) E^{2} A C<(4+\varepsilon) E^{2} A \sqrt{\frac{3 B D}{4}}, \quad 6 E A \sqrt{B}<(4+\varepsilon) \sqrt{\frac{3 D}{4}},
$$

from which it follows that

$$
E D>48(4+\varepsilon)^{-2} E^{3} A^{2} B
$$

Combining this with (6) then delivers

$$
\left(E^{2} A^{2}\right)^{2}+\gamma\left(E^{2} A^{2}\right)(E B)+3(E B)^{2}<0, \quad \gamma=6+48(4+\varepsilon)^{-2}-\frac{3}{4}(4+\varepsilon)^{2} .
$$

If $\varepsilon$ is small enough then $\gamma^{2}<12$, which renders the inequality in (7) impossible. 
Lemma 4.2. Let $A_{k} \in \mathbf{R} \backslash\{0\}$ for each $k \in T$, where $T$ is an infinite subset of $\mathbf{Z}$, and assume that $\lim _{|k| \rightarrow+\infty, k \in T}\left|A_{k}\right|=+\infty$ and

$$
M(z)=C z+R(z)+\sum_{k \in T} D_{k}\left(\frac{1}{A_{k}-z}-\frac{1}{A_{k}}\right), \quad \sum_{k \in T} \frac{D_{k}}{A_{k}^{2}}<\infty
$$

where $C \geq 0, D_{k}>0$ and $R$ is a real rational function with $R(\infty) \in \mathrm{C}$. Assume further that if $\sum_{k \in T} D_{k}<\infty$ then $R$ is constant. Then there exists $d_{1}>0$ such that, as $x \rightarrow+\infty$ with $x$ real,

$$
M^{\prime}(x) \geq \frac{d_{1}}{x^{2}}, \quad M^{\prime \prime \prime}(x)>0, \quad\left|M^{\prime \prime}(x)\right| \leq(1+o(1)) \sqrt{\frac{2}{3} M^{\prime}(x) M^{\prime \prime \prime}(x)} .
$$

Furthermore, $M$ satisfies $M(i y)=$ Ciy $+o(|y|)$ as $|y| \rightarrow+\infty$ with y real.

Proof. Let $x$ be real and tend to $+\infty$, and drop the subscript in the summation. Then

$$
M^{\prime}(x)=C+R^{\prime}(x)+\sum \frac{D_{k}}{\left(A_{k}-x\right)^{2}} \geq R^{\prime}(x)+\sum_{\left|A_{k}\right| \leq x} \frac{D_{k}}{\left(A_{k}-x\right)^{2}} \geq R^{\prime}(x)+\sum_{\left|A_{k}\right| \leq x} \frac{D_{k}}{4 x^{2}} .
$$

This yields the first inequality of (9), whether or not $\sum D_{k}<\infty$ (in which case $R^{\prime}$ vanishes identically). Moreover, $M^{\prime}$ satisfies

$$
M^{\prime}(x) \geq(1+o(1)) \sum \frac{D_{k}}{\left(A_{k}-x\right)^{2}}, \quad R^{\prime}(x)=o\left(M^{\prime}(x)\right) .
$$

Similar considerations reveal that

$$
M^{\prime \prime \prime}(x)=R^{\prime \prime \prime}(x)+\sum \frac{6 D_{k}}{\left(A_{k}-x\right)^{4}} \geq R^{\prime \prime \prime}(x)+\sum_{\left|A_{k}\right| \leq x} \frac{3 D_{k}}{8 x^{4}}
$$

and so

$$
M^{\prime \prime \prime}(x) \geq(1+o(1)) \sum \frac{6 D_{k}}{\left(A_{k}-x\right)^{4}}>0, \quad R^{\prime \prime \prime}(x)=o\left(M^{\prime \prime \prime}(x)\right) .
$$

It is easy to see from the Laurent series expansion that $R^{\prime \prime}(x)^{2}=O\left(\left|R^{\prime}(x) R^{\prime \prime \prime}(x)\right|\right)$, and so combining (10) and (11) with an application of the Cauchy-Schwarz inequality leads to

$$
\begin{aligned}
\left|M^{\prime \prime}(x)\right| & \leq\left|R^{\prime \prime}(x)\right|+\sum \frac{2 D_{k}}{\left|A_{k}-x\right|^{3}} \\
& \leq O\left(\sqrt{\left|R^{\prime}(x) R^{\prime \prime \prime}(x)\right|}\right)+2 \sum\left(\frac{\sqrt{D_{k}}}{\left|A_{k}-x\right|} \cdot \frac{\sqrt{D_{k}}}{\left|A_{k}-x\right|^{2}}\right) \\
& \leq o\left(\sqrt{M^{\prime}(x) M^{\prime \prime \prime}(x)}\right)+\sqrt{\frac{2}{3} \sum \frac{D_{k}}{\left(A_{k}-x\right)^{2}} \cdot \sum \frac{6 D_{k}}{\left(A_{k}-x\right)^{4}}} \\
& \leq(1+o(1)) \sqrt{\frac{2}{3} M^{\prime}(x) M^{\prime \prime \prime}(x)}
\end{aligned}
$$

and so (9) is proved in full. 
To prove the last assertion, let $Q$ be large and positive. Then as $|y| \rightarrow+\infty$ with $y$ real,

$$
\left|\sum_{k \in T} D_{k}\left(\frac{1}{A_{k}-i y}-\frac{1}{A_{k}}\right)\right| \leq\left|\sum_{\left|A_{k}\right|>Q} \frac{D_{k} i y}{A_{k}\left(A_{k}-i y\right)}\right|+O(1) \leq|y|\left(\sum_{\left|A_{k}\right|>Q} \frac{D_{k}}{A_{k}^{2}}+o(1)\right) .
$$

The next lemma essentially follows the local analysis of [25, Lemma 14.1].

Lemma 4.3. Let the function $L$ be non-constant and meromorphic in the plane and define $F$ by $F(z)=z-1 / L(z)$. Let $a \in \mathbf{C}$ be a zero of $L$ of multiplicity $q \in \mathbf{N}$, and let $0<\varepsilon<\pi / 16$ and $\pi / 4<\theta<3 \pi / 4$. Then the following assertions hold for all sufficiently small positive $\rho$.

There exist pairwise disjoint arcs $\lambda_{j}, j=1, \ldots, q$, each mapped by $w=L(z)$ onto the arc

$$
\Omega_{\theta, \rho}=\left\{t e^{i \theta}: 0<t<\rho\right\}
$$

and satisfying $z \rightarrow a$ as $L(z) \rightarrow 0$ on $\lambda_{j}$. Moreover, there are $q$ pairwise disjoint components $E_{j}$ of the set

$$
E=\left\{z \in \mathbf{C}: \frac{1}{2 \rho}<|F(z)|<\infty, \varepsilon<\arg F(z)<\pi-\varepsilon\right\}
$$

which satisfy $a \in \partial E_{j}$, and these may be labelled so that $\lambda_{j} \subseteq E_{j}$.

Proof. There exists $b \in \mathbf{C} \backslash\{0\}$ with

$$
L(z) \sim\left(\frac{z-a}{b}\right)^{q}, \quad F(z) \sim-\frac{1}{L(z)} \sim-\left(\frac{b}{z-a}\right)^{q}
$$

as $z \rightarrow a$. The existence for sufficiently small positive $\rho$ of $q$ pairwise disjoint $\operatorname{arcs} \lambda_{j}$ as in the lemma, as well as of $q$ pairwise disjoint components $F_{k}$ of the set $E$, with $a \in \partial F_{k}$, is standard. Since $\arg F(z)=\pi-\theta+o(1)$ as $L(z) \rightarrow 0$ with $\arg L(z)=\theta$ it follows that if $\rho$ is small enough then each $\lambda_{j}$ lies in one of the $F_{k}$ and the $\lambda_{j}$ are separated by the $q$ rays $z=a+b t e^{i 2 \pi p / q}, t \geq 0, p=0, \ldots, q-1$, as are the $F_{k}$. Hence for $\rho$ small the $F_{k}$ may be re-labelled to give $\lambda_{j} \subseteq E_{j}$.

The last two lemmas of this section are well known.

Lemma 4.4. [6] Let $1<r<R<\infty$ and let the function $g$ be meromorphic in $|z| \leq R$. Let $I(r)$ be a subset of $[0,2 \pi]$ of Lebesgue measure $\mu(r)$. Then

$$
\frac{1}{2 \pi} \int_{I(r)} \log ^{+}\left|g\left(r e^{i \theta}\right)\right| d \theta \leq \frac{11 R \mu(r)}{R-r}\left(1+\log ^{+} \frac{1}{\mu(r)}\right) T(R, g) .
$$

Lemma 4.5. [13] Let $S(r)$ be an unbounded positive non-decreasing function on $[1, \infty)$, and continuous from the right. Let $A>1, B>1$ and $G=\{r \geq 1: S(A r) \geq$ $B S(r)\}$. Then

$$
\overline{\log d e n s} G=\limsup _{r \rightarrow \infty}\left(\frac{1}{\log r} \int_{[1, r] \cap G} \frac{1}{t} d t\right) \leq\left(\frac{\log A}{\log B}\right) \limsup _{r \rightarrow \infty} \frac{\log ^{+} S(r)}{\log r} .
$$




\section{Proof of Theorem 1.2: first steps}

Throughout this section let the function $f$ be as in the hypotheses of Theorem 1.2. Then $L=f^{\prime} / f$ is transcendental and, since $f$ has finite lower order and finitely many zeros, $f$ must have infinitely many poles, all but finitely many of which are real. Thus $L$ has a Levin-Ostrovskii factorisation [34, 35]

$$
L=\frac{f^{\prime}}{f}=P \psi
$$

in which $P$ and $\psi$ are real meromorphic functions, such that $P$ has finitely many poles and $\psi$ is constructed as follows. Denote by $\alpha_{p}$ the distinct real poles of $f$, ordered so that $\alpha_{p}<\alpha_{p+1}$. For $|p| \geq p_{0}$, where $p_{0}$ is large, $\alpha_{p}$ and $\alpha_{p+1}$ are of the same sign, and there is a zero $\beta_{p}$ of $f^{\prime}$ in the interval $\left(\alpha_{p}, \alpha_{p+1}\right)$. Thus the product

$$
\psi(z)=\prod_{|p| \geq p_{0}} \frac{1-z / \beta_{p}}{1-z / \alpha_{p}}
$$

converges by the alternating series test, and satisfies

$$
0<\sum_{|p| \geq p_{0}} \arg \frac{1-z / \beta_{p}}{1-z / \alpha_{p}}=\sum_{|p| \geq p_{0}} \arg \frac{\beta_{p}-z}{\alpha_{p}-z}<\pi \quad \text { for } \quad z \in H=\{z \in \mathbf{C}: \operatorname{Im} z>0\},
$$

so that $\psi(H) \subseteq H$ and [34, Ch. I.6, Thm $\left.8^{\prime}\right]$

$$
\frac{1}{5}|\psi(i)| \frac{\sin \theta}{r}<\left|\psi\left(r e^{i \theta}\right)\right|<5|\psi(i)| \frac{r}{\sin \theta} \quad \text { for } r \geq 1, \theta \in(0, \pi) .
$$

Lemma 5.1. $P$ is a rational function and $f$ has finite order.

Proof. This is standard. The first assertion follows from (15) and the inequality

$$
T(r, P) \leq N(r, P)+m\left(r, f^{\prime} / f\right)+m(r, 1 / \psi) \leq m\left(r, f^{\prime} / f\right)+O(\log r)
$$

as $r \rightarrow+\infty$, while the second is proved by applying the argument of [3, Lemma 5.1] to $1 / f$.

Since the image of $H$ under $\log \psi$ contains no disc of radius greater than $\pi / 2$, Bloch's theorem implies that

$$
\left|\frac{\psi^{\prime}\left(r e^{i \theta}\right)}{\psi\left(r e^{i \theta}\right)}\right| \leq \frac{c_{0}}{r \sin \theta} \quad \text { for } \quad r \geq 1, \theta \in(0, \pi),
$$

with $c_{0}$ a positive absolute constant. Furthermore, $\psi$ has a representation [34]

$$
\begin{aligned}
\psi(z) & =C_{1} z+C_{2}+\sum B_{k}\left(\frac{1}{A_{k}-z}-\frac{1}{A_{k}}\right), \quad \sum \frac{B_{k}}{A_{k}^{2}}<\infty, \\
B_{k} & =-\operatorname{Res}\left(\psi, A_{k}\right)>0,
\end{aligned}
$$

where $C_{1} \geq 0, C_{2} \in \mathbf{R}$ and the $A_{k}$ are the poles of $\psi$ (all of which lie in $\mathbf{R} \backslash\{0\}$ and are poles of $f$ by the construction of $\psi$ ).

Lemma 5.2. If $P(\infty) \in \mathbf{C}$ then the hypotheses of Lemma 4.2 are satisfied with $M=L=f^{\prime} / f$ and each $D_{k}$ a positive integer.

Proof. Each $A_{k}$ in the sum in (17) is a real pole of $f$, and the residue of $L$ at $A_{k}$ is $-B_{k} P\left(A_{k}\right)$, which must therefore be a negative integer. In particular if $P(\infty) \neq 0$ 
then $P(\infty)$ is real and positive. Thus (17) and the lemma of the logarithmic derivative lead to

$$
\begin{aligned}
L(z) & =T(z)+\sum m_{k}\left(\frac{1}{A_{k}-z}-\frac{1}{A_{k}}\right), \quad \sum \frac{m_{k}}{A_{k}^{2}}<+\infty \\
m_{k} & =-\operatorname{Res}\left(L, A_{k}\right) \in \mathbf{N}
\end{aligned}
$$

where $T$ has finitely many poles and so is a real rational function. Now (17) and (18) show that the last assertion of Lemma 4.2 holds for both $M=\psi$ and $M=L-T$, which yields

$$
\begin{aligned}
T(i y) & =L(i y)+o(|y|)=P(i y)\left(C_{1} i y+o(|y|)\right)+o(|y|)=C_{3} i y+o(|y|), \\
C_{3} & =C_{1} P(\infty) \geq 0
\end{aligned}
$$

as $|y| \rightarrow+\infty$ with $y$ real. Since $T$ is a real rational function, (18) now takes the form (8), with $\sum D_{k}=\sum m_{k}=\infty$.

Proposition 5.1. Still assuming that $f$ is as in the hypotheses of Theorem 1.2, there exists $R_{1} \in(0,+\infty)$ such that $f^{\prime \prime}(x) / f(x)$ and $f^{(4)}(x) / f(x)$ are both positive or infinite for every real $x$ with $|x| \geq R_{1}$.

Proof. Let $f$ be as in the hypotheses. Because $f(z)$ may be replaced by $f(-z)$ it suffices to consider large $x$ on the positive real axis $\mathbf{R}^{+}$. The assertion concerning $f^{\prime \prime} / f$ was already proved in [31, Section 3], and that for $f^{(4)}(x) / f(x)$ will now be established by dividing into cases.

Case I. Suppose that $P(\infty) \in \mathbf{C}$. Let $x$ be real, large and positive, and assume that

$$
\frac{f^{(4)}(x)}{f(x)}=L(x)^{4}+6 L(x)^{2} L^{\prime}(x)+4 L(x) L^{\prime \prime}(x)+3 L^{\prime}(x)^{2}+L^{\prime \prime \prime}(x)
$$

is finite and non-positive. Now Lemma 5.2 shows that the hypotheses of Lemma 4.2 are satisfied with $M=L$, so write

$$
A=|L(x)|, \quad B=L^{\prime}(x)>0, \quad C=\left|L^{\prime \prime}(x)\right|, \quad D=L^{\prime \prime \prime}(x)>0 .
$$

It then follows using Lemma 4.2 that

$$
A^{4}+6 A^{2} B+3 B^{2}+D \leq\left|4 L(x) L^{\prime \prime}(x)\right|=4 A C, \quad A C>0, \quad C<\sqrt{\frac{3}{4} B D} .
$$

Applying Lemma 4.1 with $E=1$ yields a contradiction and disposes of this case.

Case II. Suppose that $P(\infty)=\infty$ and $f$ has infinitely many poles on $\mathbf{R}^{+}$. Again let $x$ be real, large and positive. Since the residue of $L=P \psi$ at a pole $A_{k}$ of $\psi$ is $-B_{k} P\left(A_{k}\right)$ by $(17)$, it follows that $P(x)$ and $P^{\prime}(x)$ are positive. Moreover, $P$ satisfies

$$
\frac{P^{(j)}(x)}{P(x)}=O\left(x^{-j}\right) \quad \text { for } j=1,2,3 .
$$

Assuming that $f^{(4)}(x) / f(x)$ is finite and non-positive, the representation (19) yields, at $x$,

$$
\begin{aligned}
F & =P^{4} \psi^{4}+6 P^{3} \psi^{2} \psi^{\prime}+\left(4 P P^{\prime \prime}+3\left(P^{\prime}\right)^{2}\right) \psi^{2}+3 P^{2}\left(\psi^{\prime}\right)^{2}+P \psi^{\prime \prime \prime} \\
& \leq 6 P^{2} P^{\prime}|\psi|^{3}+4 P^{2}\left|\psi \psi^{\prime \prime}\right|+14 P P^{\prime}\left|\psi \psi^{\prime}\right|+3 P^{\prime}\left|\psi^{\prime \prime}\right|+3\left|P^{\prime \prime}\right| \psi^{\prime}+\left|P^{\prime \prime \prime} \psi\right| .
\end{aligned}
$$


Now (17) shows that Lemma 4.2 holds with $M=\psi$ and $R$ constant, which makes it possible to write

$$
A=|\psi(x)|, \quad B=\psi^{\prime}(x) \geq \frac{d_{1}}{x^{2}}, \quad C=\left|\psi^{\prime \prime}(x)\right|, \quad D=\psi^{\prime \prime \prime}(x)>0, \quad C^{2}<\frac{3 B D}{4} .
$$

Since $4 P(x) P^{\prime \prime}(x)+3 P^{\prime}(x)^{2}$ is positive, whether or not $P^{\prime}(\infty)$ is finite, (21) now implies that

$$
\begin{aligned}
0<G & =P^{4} A^{4}+6 P^{3} A^{2} B+3 P^{2} B^{2}+P D \\
\leq J & =4 P^{2} A C+6 P^{2} P^{\prime} A^{3}+14 P P^{\prime} A B+3 P^{\prime} C+3\left|P^{\prime \prime}\right| B+\left|P^{\prime \prime \prime}\right| A,
\end{aligned}
$$

in which the variable $x$ has been suppressed for convenience. The key to this case lies in showing that (23) implies that only the first term in $J$ is significant. To this end let $\delta$ be a small positive constant and recall that $x$ is large and positive.

Suppose first that $P^{2} P^{\prime} A^{3}>\delta J$, in which case

$$
P^{4} A^{4}+P^{3} A^{2} B=O\left(P^{2} P^{\prime} A^{3}\right) .
$$

Since $P(\infty)=\infty$ and $P$ satisfies $(20)$ this leads to

$$
A=O\left(P^{\prime} / P^{2}\right) \text { and } B=O\left(P^{\prime} A / P\right)=O\left(\left(P^{\prime}\right)^{2} / P^{3}\right), \quad B=O\left(x^{-3}\right),
$$

contradicting the lower bound for $B$ in (22). Similarly, if $P P^{\prime} A B>\delta J$ then

$$
P^{3} A^{2} B+P^{2} B^{2}=O\left(P P^{\prime} A B\right),
$$

which again forces $(24)$ and yields a contradiction.

Next, if $P^{\prime} C>\delta J$ then $P D+P^{2} B^{2}=O\left(P^{\prime} C\right)$, and this implies that

$$
D=O\left(P^{\prime} C / P\right), \quad B^{2}=O\left(P^{\prime} C / P^{2}\right) .
$$

But in this case (22) yields

$$
C^{4}=O\left(B^{2} D^{2}\right)=O\left(\left(P^{\prime}\right)^{3} C^{3} / P^{4}\right), \quad C=O\left(\left(P^{\prime}\right)^{3} / P^{4}\right), \quad B^{2}=O\left(\left(P^{\prime}\right)^{4} / P^{6}\right),
$$

which by (20) gives $B^{2}=O\left(x^{-6}\right)$ and again contradicts (22).

The next case is simpler: if $\left|P^{\prime \prime}\right| B>\delta J$ then $P^{2} B^{2}=O\left(\left|P^{\prime \prime}\right| B\right)$, and so $B=$ $O\left(\left|P^{\prime \prime}\right| / P^{2}\right)$ which contradicts $(22)$ again, using $(20)$.

Finally, suppose that $\left|P^{\prime \prime \prime}\right| A>\delta J$. Then $P^{4} A^{4}+P^{2} B^{2}=O\left(\left|P^{\prime \prime \prime}\right| A\right)$ and

$$
A^{3}=O\left(\left|P^{\prime \prime \prime}\right| / P^{4}\right), \quad B^{2}=O\left(\left|P^{\prime \prime \prime}\right| A / P^{2}\right), \quad B^{6}=O\left(\left|P^{\prime \prime \prime}\right|^{4} / P^{10}\right),
$$

which, in view of $(20)$, yields $B^{6}=O\left(x^{-18}\right)$ and contradicts (22).

It now follows that (23) may be written in the form

$$
P^{4} A^{4}+6 P^{3} A^{2} B+3 P^{2} B^{2}+P D<(4+\varepsilon) P^{2} A C,
$$

in which $A, B, C, D, P$ must all be positive and $\varepsilon$ may be chosen arbitrarily small and positive, provided $x$ is large enough. Using (22) and Lemma 4.1 with $E=P$ then yields a contradiction and completes the discussion of this case.

Case III. Suppose that $P(\infty)=\infty$ and $f$ has finitely many poles on $\mathbf{R}^{+}$. In this case let $\varepsilon$ be small and positive. Then the function $h(z)=1 /(z \psi(z))$ is bounded on the rays $\arg z= \pm \varepsilon$, by (15). But $\psi$ has finitely many positive poles, and hence finitely many positive zeros, by construction. Since $\psi=L / P$ has finite order it follows using the Phragmén-Lindelöf principle that $h(z)$ is bounded as $z \rightarrow \infty$ with $|\arg z| \leq \varepsilon$. Similar considerations, starting from (16), show that $z \psi^{\prime}(z) / \psi(z)$ is 
also bounded as $z \rightarrow \infty$ with $|\arg z| \leq \varepsilon$. On recalling (20) it now follows that, as $x \rightarrow+\infty$, the term $|L(x)|$ has a positive lower bound, while

$$
\frac{L^{(j)}(x)}{L(x)} \rightarrow 0 \text { for } j=1,2,3 .
$$

This implies in view of (19) that $f^{(4)}(x) / f(x) \sim L(x)^{4}$ is positive for large $x>0$.

The proof of Proposition 5.1 is complete.

The remaining two lemmas still assume that $f$ is as in the hypotheses of Theorem 1.2.

Lemma 5.3. Let $K_{\varepsilon}=\{z \in \mathbf{C}:|z| \geq 1, \varepsilon \leq|\arg z| \leq \pi-\varepsilon\}$, where $\varepsilon$ is small and positive. Then $f$ satisfies

$$
z L(z) \rightarrow \infty \quad \text { and } \quad Q_{m}(z)=\frac{f^{(m)}(z)}{f(z)}=L(z)^{m}(1+o(1))
$$

as $z \rightarrow \infty$ in $K_{\varepsilon}$, for each $m \in \mathbf{N}$.

Proof. The first assertion is standard, and may be deduced from [26, Proof of Lemma 3] applied to $1 / f$ : see also [7, Lemma 3.15]. Indeed, if $P(\infty)=\infty$ in (14) then (15) implies immediately that $z L(z) \rightarrow \infty$ in $K_{\varepsilon}$. On the other hand, if $P(\infty) \in \mathbf{C}$ then Lemma 5.2 shows that (8) holds, with $M=L$ and each $D_{k} \in \mathbf{N}$, which yields, for $z \in K_{\varepsilon}$,

$$
\operatorname{Im} L(z) \geq \sum \frac{D_{k} \operatorname{Im} z}{\left|A_{k}-z\right|^{2}}-O\left(\frac{1}{|z|}\right) \geq\left(\frac{\sin \varepsilon}{4}\right) \sum_{\left|A_{k}\right| \leq|z|} \frac{D_{k}}{|z|}-O\left(\frac{1}{|z|}\right) .
$$

The second assertion will now be proved by induction on $m$, and is obviously true for $m=1$. Assume now that $m \in \mathbf{N}$ and that the lemma has been proved for $m$. Since $P$ is a rational function, (14) and (16) lead to

$$
\frac{z L^{\prime}(z)}{L(z)}=O(1)
$$

as $z \rightarrow \infty$ in $K_{\varepsilon}$. It may be assumed by the induction hypothesis that the estimate for $Q_{m}(z)$ in (25) holds on $K_{\varepsilon / 2}$, and so Cauchy's integral formula yields

$$
Q_{m}^{\prime}(z)=m L(z)^{m-1} L^{\prime}(z)(1+o(1))+L(z)^{m} \cdot \frac{o(1)}{|z|}=o\left(|L(z)|^{m+1}\right),
$$

as $z \rightarrow \infty$ in $K_{\varepsilon}$, using the fact that $z L(z) \rightarrow \infty$. Applying the formula $Q_{m+1}=$ $Q_{m}^{\prime}+Q_{m} L$ then completes the induction.

Lemma 5.4. Let $m \in \mathbf{N}$ and let $Q_{m}=f^{(m)} / f$. Then $f$ and $L=f^{\prime} / f=Q_{1}$ satisfy

$$
m T(r, L) \sim m \bar{N}(r, f) \sim T\left(r, Q_{m}\right) \sim N\left(r, 1 / Q_{m}\right)
$$

as $r \rightarrow \infty$ in a set of logarithmic density 1 .

Proof. The first two asymptotic equalities follow from the lemma of the logarithmic derivative and the fact that each pole of $f$ is a pole of $Q_{m}$ of multiplicity $m$, while $f$ has finite order by Lemma 5.1 and finitely many zeros. To prove the last asymptotic equality let $\delta, \sigma$ and $\varepsilon$ be small positive real numbers, and write 
$h_{m}=1 / Q_{m}$. Since $h_{m}$ has finite order, Lemma 4.5 gives a positive constant $C_{1}$ such that

$$
T\left(2 r, h_{m}\right) \leq C_{1} T\left(r, h_{m}\right)
$$

for all $r$ in a set $F_{1} \subseteq[1, \infty)$ having lower logarithmic density at least $1-\delta$. Now applying Lemma 4.4 with $R=2 r$, in combination with (25) and (27), shows that

$$
T\left(r, h_{m}\right) \leq N\left(r, h_{m}\right)+O(\log r)+88 \varepsilon\left(1+\log \frac{1}{4 \varepsilon}\right) C_{1} T\left(r, h_{m}\right) \quad \text { for } r \in F_{1} .
$$

Since $\varepsilon$ may be chosen arbitrarily small this yields, for large $r \in F_{1}$,

$$
N\left(r, 1 / Q_{m}\right) \leq T\left(r, h_{m}\right) \leq(1+\sigma) N\left(r, h_{m}\right)=(1+\sigma) N\left(r, 1 / Q_{m}\right) .
$$

But $\delta$ and $\sigma$ may also be chosen arbitrarily small, and a standard argument (see for example [27, Lemma 6]) completes the proof of (26).

\section{Completion of the proof of Theorem 1.2}

Let the function $f$ be as in the hypotheses. Applying Proposition 5.1 shows that $f^{\prime \prime}$ and $f^{(4)}$ have finitely many real zeros and so the transcendental functions $Q_{m}=f^{(m)} / f$ satisfy

$$
N_{R}\left(r, 1 / Q_{m}\right)=O(\log r)
$$

as $r \rightarrow \infty$, for $m=2$ and $m=4$. Here $N_{R}(r, g)$ denotes the integrated counting function of the real poles of a meromorphic function $g$ in the plane, and $n_{R}(r, g)$ will denote the corresponding unintegrated counting function. Next, because $f^{(4)}$ has finitely many real zeros, elementary considerations show that if $\gamma_{p}$ and $\gamma_{p+1}$ are large in modulus and are real poles of $f$, such that $\gamma_{p}<\gamma_{p+1}$ and $f$ has no poles in the interval $I_{p}=\left(\gamma_{p}, \gamma_{p+1}\right)$, then $f^{(3)} / f$ has precisely one zero in $I_{p}$. This implies that

$$
n_{R}\left(r, 1 / Q_{3}\right)=\bar{n}(r, f)+O(1), \quad N_{R}\left(r, 1 / Q_{3}\right)=\bar{N}(r, f)+O(\log r)
$$

as $r \rightarrow \infty$. Combining (26) with (28) and (29) leads at once to (2), and completes the proof of Theorem 1.2.

\section{Proof of Theorem 1.3}

Let the function $f$ be as in the hypotheses of Theorem 1.3, and assume that $f^{(m)}$ and $f^{(m+2)}$ both have finitely many non-real zeros, for some integer $m \geq 0$.

Lemma 7.1. Define $L_{m}$ and $F$ by

$$
L_{m}(z)=\frac{f^{(m+1)}(z)}{f^{(m)}(z)}, \quad F(z)=z-\frac{1}{L_{m}(z)}, \quad F^{\prime}(z)=\frac{f^{(m)}(z) f^{(m+2)}(z)}{f^{(m+1)}(z)^{2}} .
$$

Then $L_{m}$ and $F$ are transcendental, but their Tsuji characteristics satisfy

$$
\mathfrak{T}\left(r, L_{m}\right)+\mathfrak{T}(r, F)=O(\log r)
$$

as $r \rightarrow \infty$. Moreover, there exist finitely many $\alpha \in \mathbf{C}$ such that $F(z)$ or $L_{m}(z)$ tends to $\alpha$ as $z$ tends to infinity along a path in $\mathbf{C} \backslash \mathbf{R}$.

For real $K>0$ and $H=\{z \in \mathbf{C}: \operatorname{Im} z>0\}$ let

$$
H_{K}=\{z \in H:|z|>K\}, \quad W_{K}=\left\{z \in H: F(z) \in H_{K}\right\} .
$$


Then there exists a large positive real number $K$ such that the inverse function $F^{-1}$ has no singular values in $H_{K}$, and $F$ maps each component of $W_{K}$ conformally onto $H_{K}$.

Proof. Observe first that $L_{m}$ and $F$ are transcendental since otherwise $f^{(m)}$ has finite order and so has $f$. Since $L_{m}$ has finitely many non-real poles, while $f^{(m)}$ and $f^{(m+2)}$ have finitely many non-real zeros, the functions $Q=1 / L_{m}$ and $Q^{\prime}-1=-F^{\prime}$ have finitely many non-real zeros. Hence a standard application of Hayman's alternative to $Q$ as in [3], with the Nevanlinna characteristic replaced by that of Tsuji, shows that $L_{m}$ and $F$ satisfy (31). This now implies in view of Lemma 2.2 the existence of finitely many $\alpha \in \mathbf{C}$ such that $F(z)$ or $L_{m}(z)$ tends to $\alpha$ as $z$ tends to infinity along a path in $\mathbf{C} \backslash \mathbf{R}$. In particular, the real meromorphic function $F$ has finitely many non-real asymptotic values, while $F^{\prime}$ has finitely many non-real zeros. This ensures the existence of $K$ as in the lemma.

Lemma 7.2. There exist $\theta \in(\pi / 4,3 \pi / 4)$ and $N_{0} \in \mathbf{N}$ with the following properties. First, the inverse function $L_{m}^{-1}$ has no singular values in $R^{+}=\left\{r e^{i \theta}: 0<r<\right.$ $\infty\}$, nor in $R^{-}=\left\{r e^{-i \theta}: 0<r<\infty\right\}$. Next, define $x$ by $x \sin \theta=K$, where the real number $K$ is as chosen in Lemma 7.1. Then there exist at most $N_{0}$ points $z$ which satisfy $L_{m}(z) \in R^{+}$and lie on the circle $S(0,2 x)$ of centre 0 and radius $2 x$.

Proof. Both assertions follow from Lemma 7.1 and the fact that $L_{m}$ is transcendental.

Lemma 7.3. Let $D$ be a component of the set $W_{K}$, and let $a \in \partial D$ be a zero of $L_{m}$. Then $a$ is unique, and for all sufficiently small positive $\rho$ there exists at most one path lying in $D$ and tending to $a$ which is mapped by $L_{m}$ onto the arc $\Omega_{\theta, \rho}$ defined by (12).

Proof. Choose $\varepsilon$ with $0<\varepsilon<\pi / 16$ and let $\rho$ be small and positive. Then $D$ contains precisely one component $E^{\prime}$ of the set $E$ as defined by (13), since $F$ maps $D$ conformally onto $H_{K}$, and $a \in \partial E^{\prime}$. This implies that $a$ is unique, and the second assertion follows from Lemma 4.3.

The next lemma is a refinement of [33, Lemma 3.1].

Lemma 7.4. There exists a positive integer $N_{1}$ with the following property. Let $D$ be a component of $W_{K}$. Then there exist at most $N_{1}$ components $\Gamma$ of $\partial D$ with $\Gamma \subseteq H$.

Proof. Let $\Gamma \subseteq H$ be a component of $\partial D$. If $\Gamma$ contains a Jordan curve $\Gamma_{1}$ then as $z$ describes $\Gamma_{1}$ the image $F(z)$ must describe the whole extended boundary $\partial_{\infty} H_{K}=\partial H_{K} \cup\{\infty\}$, since $F$ is univalent on $D$; but this gives $\partial D=\Gamma_{1}$, from which the assertion of the lemma trivially follows. Assume henceforth that $\Gamma$ contains no Jordan curve; then $\Gamma$ is a simple curve which goes to infinity in both directions. As $z$ tends to infinity in either direction along $\Gamma$, the image $F(z)$ travels monotonely along $\partial_{\infty} H_{K}$ and is forced to tend to an asymptotic value $\alpha$ of $F$. Since $\Gamma$ lies in $H$, Lemma 7.1 implies that there are at most $M_{0} \in \mathbf{N}$ possible values $\alpha$, where $M_{0}$ is independent of $D$. Because $F$ is univalent on $\partial D$, there are no more than $2 M_{0}$ possible components $\Gamma$. 
The transcendental function $L=f^{\prime} / f$ again has a Levin-Ostrovskii factorisation (14), in which $P$ and $\psi$ are real meromorphic functions and $P$ has finitely many poles $[34,35]$. In this setting, if $f$ has finitely many poles then $\psi=1$, while if $f$ has infinitely many poles then $\psi$ is an infinite product constructed exactly as in Section 5 . In either case $\psi$ satisfies (15) and (16).

Lemma 7.5. The function $P$ is transcendental.

Proof. This follows from the argument of [3, Lemma 5.1] applied to $1 / f$.

Lemma 7.6. Let the central index $N(r)$ of $P$ and the set $E_{1}$ be as in Lemma 3.1. Then for large $r \in[1, \infty) \backslash E_{1}$ there exists $z_{0}$ with $\left|z_{0}\right|=r$ and with the following property. Let

$$
Q_{r}=\left\{z \in \mathbf{C}:\left|\log \frac{z}{z_{0}}\right| \leq N(r)^{-3 / 4}\right\} .
$$

Then $L_{m}(z)$ is large for all $z \in Q_{r}$ and satisfies there

$$
L_{m}(z) \sim L(z) \sim \psi\left(z_{0}\right) P\left(z_{0}\right)\left(\frac{z}{z_{0}}\right)^{N(r)} .
$$

Furthermore, $z_{0}$ may be chosen so that the set $Q_{r}$ is contained in a component $D$ of $W_{K}$.

Proof. Apply Lemma 3.1 to the transcendental function $P$ : since $P$ is real it may be assumed that $\operatorname{Im} z_{1} \geq 0$ in Lemma 3.1. This makes it possible to choose $z_{0}$ with

$$
\left|z_{0}\right|=r, \quad N(r)^{-2 / 3} \leq \arg z_{0} \leq \pi-N(r)^{-2 / 3},
$$

such that $\left|P\left(z_{0}\right)\right| \sim M(r, P)$ and

$$
P(z) \sim P\left(z_{0}\right)\left(\frac{z}{z_{0}}\right)^{N(r)} \text { and } \frac{P^{(j)}(z)}{P(z)} \sim \frac{N(r)^{j}}{z^{j}}
$$

for $j=1, \ldots, m+1$ and all $z \in Q_{r}$. In view of (16) this yields, for $z \in Q_{r}$,

$$
L(z) \sim \psi\left(z_{0}\right) P\left(z_{0}\right)\left(\frac{z}{z_{0}}\right)^{N(r)}
$$

and (4) implies, with $c_{j}$ denoting positive constants which are independent of $r$ and $z_{0}$, that

$$
|L(z)| \geq \frac{c_{1} M(r, P)}{r N(r)^{2 / 3} \exp \left(N(r)^{1 / 4}\right)} \geq M(r, P)^{1 / 2} \exp \left(N(r)^{1 / 4}\right) .
$$

Now for $j=1, \ldots, m+1$ a standard formula gives

$$
\frac{f^{(j)}}{f}=L^{j}+P_{j}[L]
$$

in which $P_{j}[L]$ is a polynomial in $L, L^{\prime}, \ldots, L^{(j-1)}$ of total degree at most $j-1$. Thus (16), (34), (35), (37), (38), Cauchy's estimate for derivatives and Leibnitz' rule yield

$$
\frac{\psi^{(j)}(z)}{\psi(z)}=O\left(N(r)^{2 j / 3}\right), \quad \frac{L^{(j)}(z)}{L(z)}=O\left(N(r)^{j}\right) \quad \text { and } \quad \frac{f^{(j)}(z)}{f(z)} \sim L(z)^{j}
$$

for $z \in Q_{r}$ and $j=1, \ldots, m+1$, which on combination with (30) and (36) establishes (33). 
It remains to show that $Q_{r}$ is contained in a component $D$ of the set $W_{K}$. But this follows from the fact that on $Q_{r}$ both $z$ and $L_{m}(z)$ are large while $\operatorname{Im} z \geq$ $c_{2} r N(r)^{-2 / 3}>\left|1 / L_{m}(z)\right|$.

Lemma 7.7. Let $N_{2}$ be a large positive integer. Then there exist $S>0$ and pairwise distinct points $w_{j}$, for $j=1, \ldots, 4 N_{2}$, each of large modulus and satisfying $L_{m}\left(w_{j}\right)=S e^{i \theta}$, and all lying in the same component $D$ of the set $W_{K}$.

Proof. Apply Lemma 7.6 with $r \in E_{1}$ large. On $Q_{r}$ write

$$
\zeta=\log \frac{z}{z_{0}}, \quad g(\zeta)=\log L_{m}(z)=N(r) \zeta+\log \left(\psi\left(z_{0}\right) P\left(z_{0}\right)\right)+o(1),
$$

using (33). The image of $Q_{r}$ under $\zeta$ contains the open disc $V$ of centre 0 and radius $N(r)^{-3 / 4}$. Thus $g^{\prime}(0) \sim N(r)$ and Bloch's theorem implies that $g(V)$ contains a disc of radius $N(r)^{1 / 8}$. This gives $4 N_{2}$ distinct points $w_{j} \in Q_{r}$, all satisfying $L_{m}\left(w_{j}\right)=S e^{i \theta}$ for some large positive $S$.

The proof of Theorem 1.3 will now be completed using a combination of ideas from $[32,33]$.

Lemma 7.8. For $j=1, \ldots, 4 N_{2}$ choose a component $\sigma_{j}$ of $L_{m}^{-1}\left(R^{+}\right)$with $w_{j} \in$ $\sigma_{j}$, where $R^{+}$is as in Lemma 7.2. Then the $\sigma_{j}$ are pairwise disjoint and each is mapped univalently onto $R^{+}$by $L_{m}$. Moreover at least $2 N_{2}$ of the $\sigma_{j}$ are such that $\sigma_{j}$ lies in $H_{2 x} \cap D$ and has the following property: as $w \rightarrow 0$ on $R^{+}$the pre-image $z=L_{m}^{-1}(w) \in \sigma_{j}$ tends to infinity in $D$.

Proof. The first two assertions follow from the fact that $L_{m}^{-1}$ has no singular values on $R^{+}$, by the choice of $\theta$. Since the $w_{j}$ and $N_{2}$ are large, it follows from Lemma 7.2 that at least $3 N_{2}$ of the $\sigma_{j}$ are contained in $H_{2 x}$ as defined by (32): let $z$ lie on one of these $\sigma_{j}$. Then $F(z) \in H$ by $(30)$, since $L_{m}(z) \in H$. Moreover if $\left|L_{m}(z)\right| \geq 1 / x$ then

$$
|F(z)|>2 x-x=x>K,
$$

while if $\left|L_{m}(z)\right|=r<1 / x$ then

$$
|F(z)| \geq \operatorname{Im} F(z) \geq \frac{\sin \theta}{r}>x \sin \theta=K .
$$

Thus, in either case, $z$ remains in $D$ for all $z$ on at least $3 N_{2}$ of the $\sigma_{j}$, and these have the property that, as $L_{m}(z) \rightarrow 0$ on $\sigma_{j}$, the pre-image $z$ tends either to infinity or to a zero $a$ of $L_{m}$ on $\partial D$; applying Lemma 7.3 now shows that $z$ must tend to infinity for all but one of these $j$.

After re-labelling if necessary it may be assumed that $\sigma_{j}$ satisfies the conclusions of Lemma 7.8 for $j=1, \ldots, 2 N_{2}$. For these $j$ let $\sigma_{j}^{\prime}$ be the maximal subpath of $\sigma_{j}$ on which $\left|L_{m}(z)\right| \leq S$. These paths $\sigma_{j}^{\prime}$ can then be extended to simple paths $\tau_{j}$ in $D$ which are pairwise disjoint apart from a common starting point $z^{*} \in D$. Since $N_{2}$ is large, applying Lemma 7.4 gives at least $N_{2}$ pairwise disjoint domains $\Omega_{k}$, each bounded by two of the $\tau_{j}$, and so by two of the $\sigma_{j}^{\prime}$ and a bounded simple path $\lambda_{k} \subseteq D$, such that $\Omega_{k} \subseteq D$ and the closure of $\Omega_{k}$ does not meet $\partial D$. Because $F$ has no poles in $D$, there exists a small $r_{k}>0$ such that $\left|L_{m}(z)\right| \geq r_{k}$ on $\lambda_{k}$.

For each $\Omega_{k}$ apply Lemma 7.1 to determine $P_{k} \in\left(0, r_{k}\right)$ such that the circle $S\left(0, P_{k}\right)$ contains no critical values of $L_{m}$ and no $\alpha \in \mathbf{C}$ such that $L_{m}(z)$ tends to $\alpha$ as $z$ to infinity along a path in $D$. Choose $u_{k} \in \partial \Omega_{k}$ with $L\left(u_{k}\right)=P_{k} e^{i \theta}$; here $u_{k}$ 
can be chosen to lie on one of the $\sigma_{j}^{\prime}$. Now continue $z=L_{m}^{-1}(w)$ along $S\left(0, P_{k}\right)$ in the direction which takes $z$ into $\Omega_{k}$. By the choice of $P_{k}$ and the fact that $\Omega_{k} \subseteq D$ this brings $z$ to $v_{k} \in \Omega_{k}$ with $L_{m}\left(v_{k}\right)=P_{k} e^{-i \theta}$. The choice of $\theta$ in Lemma 7.2 then makes it possible to continue $L_{m}^{-1}(w)$ along the half-ray $w=t e^{-i \theta}$, in the direction of decreasing real $t$, so that $z=L_{m}^{-1}(w)$ starts at $v_{k}$ and stays in $\Omega_{k} \subseteq D$. Since $L_{m}(z) \neq 0$ on $D$ this gives a path tending to infinity in $\Omega_{k}$ on which $L_{m}(z)$ tends to 0 with $\arg L_{m}(z)=-\theta$. Thus there exists an unbounded component $V_{k}$ of the set $\left\{z \in \mathbf{C}: \operatorname{Im}\left(1 / L_{m}(z)\right)>2 / P_{k}\right\}$, with $V_{k} \cup \partial V_{k} \subseteq \Omega_{k} \subseteq D$. Writing

$$
u_{k}(z)=\operatorname{Im} \frac{1}{L_{m}(z)} \quad\left(z \in V_{k}\right), \quad u_{k}(z)=\frac{2}{P_{k}} \quad\left(z \notin V_{k}\right),
$$

then makes $u_{k}$ non-constant and subharmonic in the plane, because $L_{m}(z) \neq 0$ on $D$. Since there are at least $N_{2}$ of these components $V_{k}$, with disjoint closures, and since $N_{2}$ is large, applying the Phragmén-Lindelöf principle [15] gives a large $z$ in one of the $V_{k}$, and so in $D$, with

$$
\operatorname{Im} \frac{1}{L_{m}(z)}>|z|^{2}
$$

and hence $\operatorname{Im} F(z)<0$ by (30), which is plainly a contradiction.

\section{Proof of Theorem 1.5}

To prove Theorem 1.5, let $g$ be as in the hypotheses, and write

$$
f=g^{(m)}, \quad L=\frac{f^{\prime}}{f}, \quad F(z)=z-\frac{1}{L(z)}, \quad F^{\prime}=\frac{f f^{\prime \prime}}{\left(f^{\prime}\right)^{2}} .
$$

It may be assumed that $L$ and $F$ are transcendental. Let $k=\min \{n, m\}$ and $h=g^{(k)}$. Then $\max \{n, m+2\} \geq k+3$ and there exists $j \geq 3$ such that $h$ and $h^{(j)}$ have finitely many non-real zeros. Thus Frank's method [9] (see also [10]), using Tsuji functionals in place of the standard Nevanlinna functionals, yields $\mathfrak{T}\left(r, h^{\prime} / h\right)=O(\log r)$ as $r \rightarrow$ $\infty$. Since $m \geq k$ it then follows that $\mathfrak{T}(r, L)=O(\log r)$ as $r \rightarrow \infty$, by standard properties of the Tsuji characteristic. Because $F$ and $1 / L$ have finitely many poles in the open upper half-plane $H$, Lemma 2.2 shows that there exist finitely many $\alpha \in \mathbf{C}$ such that $F(z)$ or $L(z)$ tends to $\alpha$ as $z$ tends to infinity along a path in $\mathbf{C} \backslash \mathbf{R}$. This implies at once that $F$ and $L$ satisfy the conclusions of Lemmas 2.1 and 3.2 of [33]. Since those two lemmas were the only steps in the proof of Theorem 1.4 in [33] which required the hypothesis that $f^{\prime} / f$ has finite lower order (see [33, Remark 3.3, p. 247] for an explicit confirmation of this observation), and since $f$ and $f^{\prime \prime}$ have finitely many non-real zeros, the remainder of the proof of Theorem 1.5 now follows exactly that of Theorem 1.4.

\section{References}

[1] Bergweiler, W., and A. Eremenko: On the singularities of the inverse to a meromorphic function of finite order. - Rev. Mat. Iberoamericana 11, 1995, 355-373.

[2] Bergweiler, W. and A. Eremenko: Proof of a conjecture of Pólya on the zeros of successive derivatives of real entire functions. - Acta Math. 197, 2006, 145-166.

[3] Bergweiler, W., A. Eremenko, and J. K. Langley: Real entire functions of infinite order and a conjecture of Wiman. - Geom. Funct. Anal. 13, 2003, 975-991. 
[4] Craven, T., G. Csordas, and W. Smith: Zeros of derivatives of entire functions. - Proc. Amer. Math. Soc. 101, 1987, 323-326.

[5] Craven, T., G. Csordas, and W. Smith: The zeros of derivatives of entire functions and the Pólya-Wiman conjecture, - Ann. of Math. (2) 125, 1987, 405-431.

[6] Edrei, A., and W. H. J. Fuchs: Bounds for the number of deficient values of certain classes of meromorphic functions. - Proc. London Math. Soc. (3) 12, 1962, 315-344.

[7] Edwards, S., and S. Hellerstein: Non-real zeros of derivatives of real entire functions and the Pólya-Wiman conjectures, - Complex Var. Theory Appl. 47, 2002, 25-57.

[8] Eremenko, A.: Meromorphic functions with small ramification, - Indiana Univ. Math. J. 42, 1994, 1193-1218.

[9] Frank, G., W. Hennekemper, and G. Polloczek: Über die Nullstellen meromorpher Funktionen und deren Ableitungen. - Math. Ann. 225, 1977, 145-154.

[10] Frank, G., and J. K. Langley: Pairs of linear differential polynomials. - Analysis 19, 1999, 173-194.

[11] Gol'dberg, A. A., and I. V. OstrovskiI: Distribution of values of meromorphic functions. Nauka, Moscow, 1970 (in Russian); English transl., Transl. Math. Monogr. 236, Amer. Math. Soc., Providence, 2008.

[12] Hayman, W. K.: Meromorphic functions. - Clarendon Press, Oxford, 1964.

[13] Hayman, W.K.: On the characteristic of functions meromorphic in the plane and of their integrals. - Proc. London Math. Soc. (3) 14A, 1965, 93-128.

[14] Hayman, W. K.: The local growth of power series: a survey of the Wiman-Valiron method. - Canad. Math. Bull. 17, 1974, 317-358.

[15] Hayman, W. K.: Subharmonic functions. Volume 2. - Academic Press, London, 1989.

[16] Hellerstein, S., and J. Williamson: Derivatives of entire functions and a question of Pólya. - Trans. Amer. Math. Soc. 227, 1977, 227-249.

[17] Hellerstein, S., and J. Williamson: Derivatives of entire functions and a question of Pólya, II. - Trans. Amer. Math. Soc. 234, 1977, 497-503.

[18] Hellerstein, S., and J. Williamson: The zeros of the second derivative of the reciprocal of an entire function. - Trans. Amer. Math. Soc. 263, 1981, 501-513.

[19] Hellerstein, S., L.-C. Shen, and J. Williamson: Real zeros of derivatives of meromorphic functions and solutions of second order differential equations. - Trans. Amer. Math. Soc. 285, $1984,759-776$.

[20] Hinkkanen, A.: Reality of zeros of derivatives of meromorphic functions. - Ann. Acad. Sci. Fenn. Math. 22, 1997, 1-38.

[21] Hinkkanen, A.: Zeros of derivatives of strictly non-real meromorphic functions. - Ann. Acad. Sci. Fenn. Math. 22, 1997, 39-74.

[22] Hinkkanen, A.: Iteration, level sets, and zeros of derivatives of meromorphic functions. Ann. Acad. Sci. Fenn. Math. 23, 1998, 317-388.

[23] KI, H., and Y.-O. Kim: On the number of nonreal zeros of real entire functions and the Fourier-Pólya conjecture. - Duke Math. J. 104, 2000, 45-73.

[24] Kim, Y.-O.: A proof of the Pólya-Wiman conjecture. - Proc. Amer. Math. Soc. 109, 1990, $1045-1052$.

[25] Langley, J. K.: Non-real zeros of higher derivatives of real entire functions of infinite order. - J. Anal. Math. 97, 2005, 357-396.

[26] Langley, J. K.: Solution of a problem of Edwards and Hellerstein. - Comput. Methods Funct. Theory 6, 2006, 243-252. 
[27] Langley, J. K.: Meromorphic functions in the class $S$ and the zeros of the second derivative. - Comput. Methods Funct. Theory 8, 2008, 73-84.

[28] Langley, J. K.: Non-real zeros of derivatives of real meromorphic functions. - Proc. Amer. Math. Soc. 137, 2009, 3355-3367.

[29] Langley, J. K.: Non-real zeros of linear differential polynomials. - J. Anal. Math. 107, 2009, $107-140$.

[30] Langley, J. K.: Zeros of derivatives of meromorphic functions. - Comput. Methods Funct. Theory 10, 2010, 421-439.

[31] Langley, J. K.: Non-real zeros of real differential polynomials. - Proc. Roy. Soc. Edinburgh Sect. A 141, 2011, 631-639.

[32] Langley, J. K.: Non-real zeros of derivatives of real meromorphic functions of infinite order. - Math. Proc. Camb. Phil. Soc. 150, 2011, 343-351.

[33] Langley, J. K.: Zeros of derivatives of real meromorphic functions. - Comput. Methods Funct. Theory 12, 2012, 241-256.

[34] Levin, B. JA.: Distribution of zeros of entire functions. - GITTL, Moscow, 1956; 2nd English transl., AMS, Providence RI, 1980.

[35] Levin, B. JA., and I. V. OstrovskiI: The dependence of the growth of an entire function on the distribution of zeros of its derivatives. - Sibirsk. Mat. Zh. 1, 1960, 427-455; English transl., AMS Transl. (2) 32, 1963, 323-357.

[36] Pólya, G.: On the zeros of the derivatives of a function and its analytic character. - Bull. Amer. Math. Soc. 49, 1943, 178-191.

[37] Rossi, J.: The reciprocal of an entire function of infinite order and the distribution of the zeros of its second derivative. - Trans. Amer. Math. Soc. 270, 1982, 667-683.

[38] Sheil-Small, T.: On the zeros of the derivatives of real entire functions and Wiman's conjecture. - Ann. of Math. 129, 1989, 179-193.

[39] Tsuji, M.: On Borel's directions of meromorphic functions of finite order, I. - Tôhoku Math. J. 2, 1950, 97-112.

Received 13 March 2013• Accepted 22 March 2013 